\title{
Exemple historique et argumentation : autour de la reconnaissance du massacre du 17 octobre 1961
}

Historical Example and Argumentation: the Acknowledgement of the October $17^{\text {th }} 1961$ Massacre

\section{Paola Paissa}

\section{CpenEdition}

Electronic version

URL: http://journals.openedition.org/aad/2160

DOI: $10.4000 /$ aad. 2160

ISSN: $1565-8961$

\section{Publisher}

Université de Tel-Aviv

\section{Electronic reference}

Paola Paissa, «Exemple historique et argumentation : autour de la reconnaissance du massacre du 17 octobre 1961 », Argumentation et Analyse du Discours [Online], 16 | 2016, Online since 09 April 2016 connection on 23 September 2019. URL : http://journals.openedition.org/aad/2160 ; DOI : 10.4000/ aad. 2160

This text was automatically generated on 23 September 2019.

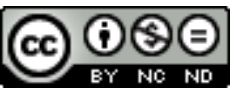

Argumentation \& analyse du discours est mis à disposition selon les termes de la licence Creative Commons Attribution - Pas d'Utilisation Commerciale - Pas de Modification 4.0 International. 


\title{
Exemple historique et argumentation : autour de la reconnaissance du massacre du 17 octobre 1961
}

\author{
Historical Example and Argumentation: the Acknowledgement of the October \\ $17^{\text {th }} 1961$ Massacre
}

Paola Paissa

1 Cet article vise à examiner la polémique qui s'est engagée dans certains blogs de lecteurs réagissant à un acte de langage institutionnel ${ }^{1}$ : le communiqué du 17 octobre 2012, par lequel la Présidence de la République a reconnu la "répression sanglante » qui, le 17 octobre 1961, avait provoqué la mort de nombreux manifestants algériens à Paris. En effet, ce jour-là (cinq mois avant les accords d'Evian), une manifestation pacifique de ressortissants algériens, organisée pour protester contre le couvre-feu imposé par le Préfet de Paris, Maurice Papon, avait déclenché une réaction violente de la police parisienne, causant entre 50 et 200 victimes (le nombre varie dans les estimations des historiens). Ce massacre, longtemps occulté de la mémoire collective des Français est, depuis 2001, commémoré par une plaque apposée par Bertrand Delanoë sur le Pont Saint-Michel.

2 Le corpus de notre étude est formé de plus de six cents interventions de lecteurs, publiées dans différents sites web, à la suite d'articles ou interviews portant sur le communiqué de $2012^{2}$. Les commentaires concernent un acte discursif qui a provoqué un retour de mémoire à propos d'un événement controversé et présentent une fréquence élevée de références à des faits historiques divers. Tout en se déployant sur des plans différents, ces références assument souvent la fonction d'un argument. C'est dans ces cas-là que nous les considérerons comme des exemples historiques (désormais $\mathrm{EH})$, l'événement historique évoqué représentant le fondement d'un raisonnement qui vise à fournir une preuve par l'exemple. Les formes que ce procédé emprunte dans les conversations numériques, ainsi que les fonctions qu'il recouvre, sont multiples. $\mathrm{Ce}$ 
tableau hétéroclite peut néanmoins se résumer dans les trois points suivants, représentant autant de parties de notre analyse :

- 1. L'EH est un élément constitutif de l'inscription discursive de l'acte accompli par le Président, qui le consigne dans le paradigme de la " repentance »;

- 2. l'EH est un élément essentiel du discours argumentatif axiologique (favorable ou défavorable) suscité par cette initiative de la Présidence ;

- 3. l'EH est un élément fondamental de la stratégie argumentative la plus fréquente dans le corpus, à savoir celle qui exploite l'argument de la direction, ou argument de «la pente glissante »(Perelman et Olbrechts-Tyteca 2008, Walton 1992, Angenot 2012)3.

\section{L'EH et le paradigme discursif de la « repentance»}

Le communiqué émis par la Présidence de la République Française le 17 octobre 2012, que nous reproduisons ci-dessous, est, somme toute, assez laconique :

Le 17 octobre 1961, des Algériens qui manifestaient pour le droit à l'indépendance ont été tués lors d'une sanglante répression. La République reconnaît avec lucidité ces faits. Cinquante et un ans après cette tragédie, je rends hommage à la mémoire des victimes.

Les quelques lignes composant le communiqué ne contiennent aucune expression relevant d'un véritable acte de "repentance». Quelques expressions pathémiques (« sanglante répression », "tragédie », « victimes ») sont utilisées pour reconnaitre le méfait, mais aucune allusion n'est faite à des regrets, ou à d'autres sentiments susceptibles d'accompagner le « repentir public $»^{4}$. En revanche, le bref texte fait appel à la « lucidité », une faculté d'ordre intellectuel qui ne relève ni du domaine éthique ni du domaine politique. Enfin, l'acte de « reconnaissance » n'est pas directement endossé par le Président, qui se borne à rendre "hommage à la mémoire des victimes », la parole performative de " reconnaître » émanant, elle, du sujet social et collectif qu'est la «République $»^{5}$. Si donc, malgré sa prudence, ce communiqué est immédiatement reçu par les opposants politiques et par les médias comme un geste de "repentance ", c'est qu'on le situe dans le droit fil d'une série de prises de position publiques et d'un groupe de lois - dites "mémorielles" - destinées à imprimer une orientation normative à la mémoire de certains événements du passé.

Dans les réactions du monde politique et de la presse, l'acte de reconnaissance voulu par Hollande est rapproché principalement de deux événements passés : 1) l'allocution de Chirac de 1995 qui, lors de la Commémoration de la rafle du Vélodrome d'Hiver, avait admis la responsabilité française dans la déportation des Juifs de France ; 2) la loi Taubira de 2001, qui reconnaît la culpabilité de la France dans la traite négrière et l'esclavage. Dans le débat sur le repentir, ces deux faits deviennent des EH qui agissent comme des embrayeurs, permettant l'insertion de l'événement de 2012 dans une classe ouverte d'événements semblables. Or, à l'évidence, cette insertion ne va pas sans soulever des questionnements : la nomination même de l'acte de Hollande suscite un débat de nature métadiscursive (convient-il de parler d'acte de « repentance " pour ce communiqué de la Présidence? Ne serait-il pas mieux d'y voir une simple « reconnaissance de la vérité historique »? Ne pourrait-on pas, en revanche, désigner cette démarche comme un " acte de réconciliation»?, etc.). A partir de la nomination de l'événement ${ }^{6}$, le champ discursif est d'emblée polarisé, la droite ayant tendance à catégoriser le geste de 2012 comme un acte de "repentance», alors que la gauche 
s'avère, à cet égard, beaucoup plus précautionneuse, le mot "repentance » étant $\mathrm{ambigu}^{7}$ et provoquant, à lui seul, la réactivation de la "mémoire polémique " (Maingueneau 1987: 167) qui a accompagné les autres occurrences de la même catégorie paradigmatique. À la fois l'événement historique de 1961 et l'initiative institutionnelle de 2012 favorisent donc des commentaires évaluatifs, portant sur l'attitude à adopter face à des épisodes du passé, qui orientent l'appréhension du présent $^{8}$.

\section{L'EH, l'argumentation et le discours axiologique autour du communiqué du 17 octobre 2012}

Pour ce qui est des positionnements axiologiques et idéologiques, on verra d'abord, brièvement, les réactions politiques que l'acte de Hollande a suscitées dans le monde politique et dans la presse, en Algérie et en France, afin de cadrer l'analyse des réactions des lecteurs.

- Les autorités politiques et la presse algérienne ne montrent qu'un froid enthousiasme pour la publication du communiqué du 17 octobre 2012, qui a été qualifié de "petit geste», voire de "geste timide" par le Président algérien Bouteflika. Les commentateurs algériens convoquent ainsi la mémoire de quelques autres événements du même genre, pour accuser la France de procéder à des reconnaissances partielles, réduisant le crime historique représenté par la colonisation et par la guerre à quelques aspects ou à quelques épisodes ${ }^{9}$. Comme l'exprime efficacement Djiali Benyoub ${ }^{10}$, l'intention française serait de "saucissonner l'histoire». Sont évoquées, pour en souligner l'insuffisance : en premier lieu, la promulgation de la loi de 1999, imposant la nomination "guerre d'Algérie " à la place des désignations officielles «événements d'Afrique du Nord » ou " opérations effectuées en Afrique du Nord », qui avaient été en vigueur jusqu'à ce moment-là, malgré leur portée généralisante et euphémique (Paissa 2012) ; en deuxième lieu, la reconnaissance des massacres de Sétif, Guelma et Kherrata de 1945, ayant fait l'objet de déclarations jugées du côté algérien comme improvisées et incomplètes.

7 En France, les positions sont à l'évidence plus variées :

- la gauche assume une position assez homogène, saluant favorablement l'initiative de Hollande. Dans le registre de l'exemplarité historique, ce camp parvient à comparer le geste voulu par le Président aux actes de réconciliation ayant rapproché la France et l'Allemagne à la fin de la Seconde Guerre Mondiale.

- En revanche, la droite française est, dans son ensemble, ouvertement hostile à l'acte de la Présidence, avec des motivations plus ou moins nuancées. Christian Jacob qualifie carrément de « ridicule » et d'« intolérable » l'initiative de Hollande ; l'UMP s'aligne sur la position qu'avait adoptée Sarkozy dès la présidentielle de 2007, endossant ce qu'on a désigné comme l'« ethos du non-repentant" (Turbide, Laforest, Vincent 2012); François Fillon rappelle qu'il y a eu également des crimes de l'autre côté, empruntant ainsi une stratégie suivie par plusieurs blogueurs, qui énumèrent les massacres perpétrés par les Algériens, avant ou après l'Indépendance de 1962, pour en conclure que la France n'est finalement tenue à aucune reconnaissance ${ }^{11}$; Xavier Weiss, quant à lui, se demande à propos de Hollande: "Jusqu'où ira-t-il ?", mettant en branle le mécanisme de l'argument de la direction. Pour finir, Marine Le Pen reprend à son compte ce mécanisme, mettant en place le schéma argumentatif de la «pente 
glissante » dans une interrogation rhétorique qui s'appuie ironiquement sur un EH improbable. En effet, la leader du FN s'exclame, de manière tout particulièrement provocatrice : «A quand la repentance pour la Saint-Barthélemy? » Dans ce dispositif, qu'on retrouvera fréquemment dans les commentaires des lecteurs, le recours à l'EH a pour fonction de tourner en dérision l'acte de repentance.

Quant au positionnement axiologique et argumentatif des blogueurs, le discours est également dichotomisé et fort déséquilibré au point de vue quantitatif, puisque les commentaires positifs sont nettement moins nombreux que les commentaires négatifs ${ }^{12}$.

Cependant, quelle que soit l'orientation axiologique, le recours à des références historiques dans ces interventions est systématique. Selon la visée argumentative (Amossy 2000 : 24) que le discours endosse, ces références peuvent assumer la fonction d'un argument et acquérir le statut d'EH, suivant la définition que nous avons formulée dans notre introduction. Toutefois, ces EH ne sont généralement pas développés, leur rappel bref et concis suffisant à justifier l'orientation adoptée par le commentateur. La forme sous laquelle ils se présentent se réduit généralement à l'allusion, à un rapide clin d'œil permettant d'établir et d'exploiter une connivence avec le lecteur ${ }^{13}$. Dans ce qui suit, nous verrons d'abord quels EH sont le plus souvent évoqués et dans quel cadre argumentatif, pour nous concentrer ensuite sur l'EH utilisé, à l'instar de Marine Le Pen, dans l'argumentation de la " pente glissante ».

\subsection{Positionnements axiologiques et idéologiques des blogueurs : les choix des $\mathrm{EH}$}

\subsubsection{Les EH dans les commentaires positifs}

Plus rares que les négatives, les réactions positives au communiqué du 17 octobre 2012 peuvent s'appuyer sur la situation sociopolitique internationale ou sur le contexte historique national. Sur le plan international, l'enjeu de la crédibilité de la France en tant que "patrie des droits de l'Homme » est souvent allégué, afin de souligner qu'il est possible de reconnaitre ses propres responsabilités historiques sans pour autant affaiblir l'ethos national et le rôle géopolitique traditionnel. Cette position, qui se rattache à la "rhétorique du juge-pénitent ${ }^{14}$, est illustrée dans un commentaire comme le suivant :

1. Qu'il y a-t-il de mal à reconnaitre les erreurs du passé ? Le fait que nous passions encore sous silence des évènements douloureux de notre passé ne nous honore pas. Bien au contraire, car on est alors peu crédibles à l'international quand on se proclame patrie des droits de l'homme. ${ }^{15}$ (Cirta, blog « francetvinfo », 20/10/2012)

11 Dans ce cadre argumentatif, on met à contribution un EH majeur : celui du génocide arménien de 1915. Cet EH est exploité pour mettre au jour une possible incohérence dans la position de la France, qui ne saurait prétendre à une reconnaissance du génocide arménien perpétré par la Turquie, sans être disposée à en faire autant pour ses propres crimes coloniaux. S'appuyant sur la règle de justice (Perelman \& OlbrechtsTyteca 2008 : 294), les lecteurs mobilisent cet $\mathrm{EH}$ dans des interventions prenant l'allure de l'invective. Ainsi, un blogueur juge-t-il «hilarant que certains hurlent à l'abomination, parce que la Turquie refuse de reconnaître le génocide arménien ", alors qu'un autre constate, à propos de l'UMP, que « c'est plus facile de demander aux Turcs de reconnaitre ses crimes que d'admettre les notres... ${ }^{16} »$. 
12 Sur le plan national, par contre, ce sont les actes de répression violente précédant l'éclatement de la guerre, et tout particulièrement les massacres du Constantinois de 1945, qui jouent le rôle d'EH. Là aussi, on applique la règle de justice, afin de faire jouer à ces événements la fonction d'arguments, pour réclamer la reconnaissance et la nomination officielle de ces méfaits comme des massacres, voire des « crimes contre l'humanité ", à l'instar du massacre du 17 octobre 1961. Autrement dit, ces EH

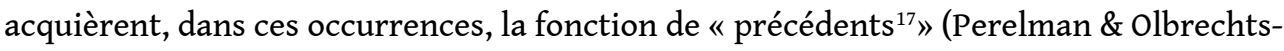
Tyteca $2008: 144$ et passim).

2. Maintenant, il ne reste plus qu'à faire la même chose à propos des massacres de Sétif et Guelma de mai 1945, alors que la France célébrait la capitulation nazie (Juan Manuel Cuesta, blog « Le Monde », 20/10/2012).

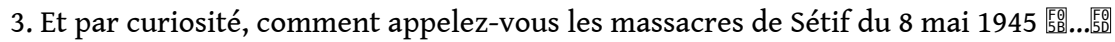
qui firent 45000 morts? 留...硻 Aujourd'hui, ce serait qualifié de crime contre l'humanité et ses auteurs jugés par la Cour de Justice Internationale de La Haye (chtiquicourt, blog «francetvinfo », 20/10/2012).

$13 \mathrm{Au}$ sein de cette polémique, l'absence d'argumentations évoquant les conditions objectives du conflit et, en premier lieu, la disproportion des rapports de force entre la France et l'Algérie, mérite d'être soulignée ${ }^{18}$. En effet, dans les conversations numériques consultées, nous n'avons repéré qu'une seule intervention qui, à partir du constat de la dissymétrie des parties dans un conflit de décolonisation (cf. Pelinka 2009), en vient à mettre en cause sa catégorisation de «guerre». Ce commentaire, brutalement rejeté par les autres intervenants, s'appuie lui aussi sur l'EH du génocide arménien, afin de suggérer une équivalence du statut victimaire des Arméniens et des Algériens, concluant à la nécessité de qualifier la guerre d'Algérie, dans son ensemble, de " génocide » :

4. On ne peux pas parler de guerre en Algérie, parce que l'Algérie ne possédait pas d'armée !!!! l'armée française s'en est pris à la population !!!!! c'est plutôt un génocide !!!! Il faut que les français le reconnaissent; surtout depuis que la France qualifie la tragédie arménienne de génocide !!!! (Mahmoud, blog "Egalité et Réconciliation », $25 / 10 / 2012)$

Dans quelques cas, à vrai dire fort exceptionnels, on convoque également la mémoire de Vichy: le soutien majoritaire accordé au régime collaborationniste est mentionné, dans ces cas-ci, pour demander que la condamnation des responsabilités coloniales soit plus nette et moins tardive que celle du pétainisme. Dans le commentaire 5, l'EH du génocide arménien dérive vers le souvenir de l'Occupation. La "France de Vichy" devient alors un EH répulsif, représentant la persévérance dans la non-reconnaissance et l'oubli :

5. Lorsqu'on entend les réactions de certains hommes politiques de droite, on comprend mieux l'entêtement des autorités turques à nier le génocide arménien. Certes rien de comparable entre ces 2 évènements, mais [...] combien de décennies avant de reconnaître que la France de Vichy a bel et bien été une réalité soutenue par une majorité de français (Enervé, blog « Le Monde », 18/10/2012).

\subsubsection{Les EH dans les commentaires négatifs}

Les réactions défavorables au geste de Hollande - nettement plus nombreuses présentent aussi une plus grande variété de formes et de stratégies argumentatives. Tous les traits du discours polémique (Amossy 2014) y sont réunis : dichotomisation, polarisation, tripartition de la structure actancielle, disqualification de la partie adverse, fréquent recours à l'argument ad personam, etc. Pour ce qui est du choix des 
$\mathrm{EH}$, deux figures centrales structurent l'argumentation opposée à la reconnaissance du massacre du 17 octobre 1961 : la négation de l'événement historique en lui-même et la négation de la responsabilité française.

Malgré le travail des historiens, qui divergent sur le nombre des victimes des affrontements du 17 octobre 1961, mais s'accordent désormais sur la dénomination de "massacre", un certain nombre de commentaires expriment soit la négation totale de l'événement (cf. Wodak 2006, Hansson 2015), soit le refus de sa catégorisation sous la « description » (Quéré 1994) de " massacre ».

Généralement, les interventions désavouant le massacre de 1961 évoquent le contexte général de l'époque, afin de minimiser la portée de l'événement et de le faire apparaître comme un accident parmi d'autres, dans un conflit ayant évolué en guerre civile de part et d'autre de la Méditerranée. Cette dynamique argumentative peut être mise en relation avec la stratégie de l'énumération des méfaits accomplis dans un camp comme dans l'autre, mentionnée en 2.1 à propos de la position de Fillon, par ailleurs commune à plusieurs hommes politiques de droite. La négation du crime et l'impossibilité de la réconciliation est justifiée, dans ces commentaires, par l'évocation des tueries dont s'est rendue coupable l'Algérie envers les Pieds-Noirs ou les Harkis, depuis la Toussaint Rouge de 1945 jusqu'au massacre d'Oran de 1962, commis en violation des accords d'Evian. Les allusions à ces événements assument dans le discours la fonction d'EH, puisqu'ils illustrent les responsabilités du camp algérien et sont avancés comme preuves à l'appui de la thèse de la non-reconnaissance. Ce dispositif persuasif exploite souvent la figure de la congérie (Perelman et Olbrechts-Tyteca 2008: 237, Rabatel $2014: 55)$, c'est-à-dire qu'on procède à un inventaire jouant sur le cumul :

6. On ne peut pas parler de réconciliation tant que la vérité historique est confisquée par les vainqueurs (politiques): non il n'y a pas eu de «massacre » à Paris en Octobre 1961 (5 morts recensés), oui à Oran en juillet 1962 les pieds-noirs ont été massacrés, oui les harkis ont été victimes des vengeances du FLN et continuent de l'être aujourd'hui (ils sont interdits de séjour en Algérie). Tant que l'Algérie ne reconnaît pas ces 3 faits historiques, aucune réconciliation n'est possible (Bara, blog « Atlantico.fr », 3/11/2012).

18 Dans ce cas de figure, la mémoire relative à la guerre d'Algérie débouche sur la négation généralisée de la responsabilité de la France dans le conflit: les références à des épisodes de l'époque s'accumulent en cascade, afin de diluer la culpabilité française. Comme le montre le commentaire 7, cela peut même aboutir à un usage discutable de la stratégie du renversement des rôles ${ }^{19}$ (« victim-victimizer reversal», Hansson 2015 : 310 , Wodak $2015: 62$ ). Axé sur la confusion des positions historiques, ce procédé réunit des événements dont les statuts sont foncièrement différents (crimes de masse, attentats terroristes, représailles contre de simples citoyens), appartenant à la "mémoire épisodique " et se situant à la croisée des perspectives individuelles et collectives (Van Dijk 1987). Qui plus est, ces faits sont souvent évoqués avec d'affreux détails visant l'hypotypose (7).

7. Je passe sur les premiers tues de la guerre d'Algérie, Massacre d'el Halia, plus de 35 européens sont massacre dans d'atroces conditions par le FLN, y compris les femmes et les enfants (passons sur les details) Massacres d'Algériens a Melouza, Wagram et ailleurs par le FLN Bombes a Alger, milk bar et autres. Européens et Algériens enlevés tortures et massacres dans des conditions bestiales. Massacres et disparitions d'Oran, personnes égorgées, pendues aux étals de boucher etc Massacre de 80000 harkis et de leur familles. Et c'est a nous de demander pardon, mais ce n'est même pas pensable (enzo31311, blog « Atlantico.fr », 3/11/2012). 

abusent également de nombreux hommes politiques, va ainsi à l'encontre du « topos de l'ethos différent " (Eggs $2014:$ 149), interdisant de mettre sur le même plan la victime et le bourreau (le colonisé et le colonisateur, en l'occurrence).

La stratégie de la négation du drame du 17 octobre 1961 et du refus d'admettre la responsabilité française peut aussi, quoique de manière plus exceptionnelle, quitter le cadre national et s'appuyer sur des événements appartenant à la mémoire collective internationale. Afin de nier le massacre parisien de 1961, certains lecteurs vont jusqu'à formuler l'hypothèse d'une falsification médiatique des faits. Des cas éclatants de manipulation de la réalité factuelle, ayant connu un retentissement international, comme le faux charnier de Timisoara (1989) ou l'affaire des couveuses de Koweit-City (1990) acquièrent alors la fonction d'EH, qui sont exploités pour émettre l'hypothèse d'une fraude :

8. Bernard Lugan l'a bien écrit hier sur son blog; Les massacres du 17 octobre 1961 sont une falsification de l'Histoire de l'ordre de Timisoara et des couveuses de Koweit-city. Honte à cette gauchiasse de flan-flan pour oser trainer la France dans la boue ainsi (Denis Josière, « Le Figaro », 18/10/2012).

Pour rester dans le contexte international, les blogueurs peuvent également évoquer la conduite d'autres pays colonisateurs, pour affirmer l'inopportunité de la «mode française de la repentance ». Ils proposent, ainsi, des parallèles avec des situations historiques disparates, donnant lieu à un emploi de l'EH qu'on pourrait considérer comme fallacieux ${ }^{20}$. On souligne, par exemple, le cas de l'Angleterre, qui n'a jamais formulé d'excuses pour la colonisation de l'Inde, ou encore celui des Etats-Unis auxquels, selon plusieurs lecteurs, on ne saurait demander de se repentir d'avoir décimé les Amérindiens, ou d'avoir provoqué les catastrophes atomiques au Japon à la fin de la Seconde Guerre mondiale. L'énoncé 9 montre clairement la facilité avec laquelle on met dans le même sac, en position d'EH à valeur d'argument, des événements foncièrement différents, depuis le Vél d'Hiv jusqu'à la Guerre du Golfe, tout en proposant de longs inventaires, souvent fort approximatifs du point de vue historique, qui sont déroulés sur un ton effronté et insolent :

9. Marre de la culpabilité perpétuelle de la france et de ces élites à la solde de l'étranger! "vélodrome d'hiver » "massacre d'indépendantistes algériens » etc ..STO0000OP! La coupe est pleine! Allez du coté de chez l'oncle sam et demandez aux ricains de se repentir pour le massacre des peaux-rouges, des deux bombardements atomiques sur Hiroshima et Nagasaki, de l'esclavage des noirs aux États-Unis, des centaines de milliers de civils irakiens massacrés... Nous on a donné! Ou alors mondialisez toutes les repentances et les opérations Kleenex (Godefroi de Javron, blog « Egalité et Réconciliation », 25/10/2012).

\section{L'EH dans l'argument de la " pente fatale »}

Cependant, la stratégie qui exploite de la manière la plus efficace l'EH est l'argument dit de la « pente fatale » ou de la " pente glissante, savonneuse ». Il s'agit d'un dispositif argumentatif qui met en scène la partie adverse comme si elle était engagée dans un chemin sans retour, correspondant au cliché discursif «jusqu'où arrivera-t-on? » De plus, comme c'est le cas dans notre corpus, cet argument recourt volontiers à des hyperboles ${ }^{21}$, déployées sur le ton du défi, de l'affrontement direct, suivant le moule «Pourquoi pas X, tant qu'on y est? »Comme nous l'avons vu, c'est Marine Le Pen qui a

Argumentation et Analyse du Discours, 16 | 2016 
recours à ce type de mécanisme argumentatif, ainsi qu'au registre de l'attaque ironique et arrogante, lorsqu'elle se demande " A quand la repentance pour la SaintBarthélemy? " Avant de voir l'utilisation de ce schéma auprès des blogueurs, une considération s'impose sur le choix de Marine Le Pen de se référer justement à l'EH de la Saint-Barthélemy. Ce choix n'est, à mon sens, pas anodin : bien au contraire, comme cela arrive souvent dans le langage de la droite (Wodak 2015), il est provocateur pour deux raisons au moins. Premièrement, parce que, pour souligner la gravité de la répression du 17 octobre 1961, la presse algérienne et internationale avait effectivement eu recours, à l'époque des faits, à des expressions telles qu'« une SaintBarthélemy des musulmans » ou "une Saint-Barthélemy moderne ", issues d'une analogie avec le carnage commis contre les protestants en 1572, alors qu'en France, la désignation des faits de 1961 à travers la mémoire de la Saint-Barthélemy ne semble pas être attestée (il est d'ailleurs fort douteux qu'elle puisse être facilement reçue). Deuxièmement, parce que, en revanche, le nom «Saint-Barthélemy » représente en France le prototype, le parangon antonomasique du " massacre ». En effet, il s'agit d'un nom d'événement qui s'est banalisé dans cet emploi, pouvant même donner lieu à des usages figuraux, tels que "s'égorger façon Saint-Barthélemy ", ou encore provoquer, par une réforme des retraites, « une Saint-Barthélemy sociale ». La décision d'utiliser le nom prototypique d'un massacre pour ridiculiser la reconnaissance d'une tuerie longtemps refoulée et occultée, qu'une frange non négligeable de Français se refuse encore à considérer comme un massacre, répond donc à une indéniable stratégie polémique de provocation. Cependant, ce que les lecteurs mettent en relief lorsqu'ils commentent la petite phrase de Marine Le Pen, c'est moins l'EH qu'elle mobilise, que le dispositif argumentatif qu'elle exploite. En effet, les lecteurs réagissant à la position de Marine Le Pen, mentionnent rarement la référence à la Saint-Barthélemy. S'il est vrai que, lorsqu'ils le font, ils renchérissent en détails cyniques, comme dans le commentaire suivant :

10. «Eh bien soit! Qu'on les tue! Mais qu'on les tue tous! Qu'il n'en reste plus un pour me le reprocher!» Massacre de la Saint-Barthélemy. La fameuse phrase prononcée par le roi, le soir du 23 août. Il se serait écrié de colère, sous les conseils répétitifs de sa mère, excédé ( $\mathrm{Mr}$ pomme de terre, blog "Égalité et Réconciliation », $25 / 10 / 2012)$

il est aisé de constater que les blogueurs choisissent le plus fréquemment d'autres caslimite, censés être représentatifs d'une dérive qui entraînerait inévitablement la France vers une issue honteuse et risible. S'ils adoptent volontiers l'argument de la pente glissante, les lecteurs abondent dans le sens de l'absurdité des EH mobilisés, au point de faire basculer cette stratégie argumentative vers l'argument ad ridiculum ${ }^{22}$. Une sorte d'effet de mimétisme discursif se produit ainsi, sans qu'on puisse établir si c'est le public qui imite le discours politique (de Marine Le Pen, en l'occurrence), ou si c'est le discours politique qui court après le discours du public. Quoi qu'il en soit, les EH se multiplient et s'inscrivent dans des configurations caractérisées par une tendance à l'accumulation énumérative et par la production d'un climax paradoxal. En outre, dans les nombreux cas attestés dans notre corpus, on peut constater la réitération du même moule pragmatico-énonciatif. Comme le montrent les énoncés rapportés ci-dessous, la modalité préférée est celle de la bravade verbale, débouchant sur l'interrogation, un mécanisme énonciatif très contraignant pour l'énonciataire, à qui on impose son propre point de vue (désormais, PDV: cf. Rabatel 2005) à travers le mouvement montant et ouvert de la forme interrogative : 
11. Jusqu'à quand cette nouvelle mode va-t-elle nous entraîner ? Et Rome, donc ? (Guff, blog « Atlantico.fr », 3/11/2012);

12. En effet, à quand les dédommagements pour les massacres de Jules César, des Francs ou des Vikings ? (ChoCo, blog « Egalité et Réconciliation », 25/10/2012) ;

13. Faut-il aussi faire repentance pour avoir « bousté » hors de chez nous les arabes à POITIERS ???? (fopapousser, blog « Atlantico.fr », 3/11/2012) ;

14. Pourquoi ne pas remonter jusqu'à Louis XIV ou Charlemagne quand on y est? (ylrug, blog « Le Figaro », 26/10/2012);

15. Allons-nous remonter jusqu'aux Chouans? (Bruce L., blog «Le Figaro", $18 / 10 / 2012)$

Pour ce qui est du référent historique des $\mathrm{EH}$, on peut souligner des différences, déterminées à la fois par la perspective temporelle et par ce qu'on pourrait appeler, suivant Perelman (1970 : 365), le « coefficient d'importance » d'un événement pour une collectivité. Ces différences ont des répercussions de taille sur la dynamique argumentative. En effet, la distance historique s'avère proportionnelle à la charge dérisoire et polémique du schéma de la "pente fatale»: lorsque l'éloignement temporel est le plus grand, avec les références à Rome, à Jules César, à Charlemagne, etc. (ex.11-15), l'intention ironique est manifeste et indiscutable. Dans ces contextes, l'argument de la direction aboutit à un anéantissement de la question problématique, qui sombre complètement dans le ridicule. En revanche, au fur et à mesure que l'on se rapproche du présent et que l'EH choisi devient plus plausible, la vigueur polémique augmente et la stratégie mise en place s'avère plus insidieuse : en effet, en mobilisant des EH plus actuels, s'accroît leur capacité à diviser les communautés de mémoires. Au lieu de servir «l'homonoïa », sur laquelle se fonde en général l'emploi des exemples (Herman 2011), ceux-ci suscitent un sentiment d'«héteronoïa». L'Occupation allemande constitue, dans ces occurrences, l'EH privilégié, tant pour manifester l'orgueil national (16), que pour avancer l'hypothèse d'une supériorité de la France sur l'Algérie (17) :

16. A quand des excuses de la France pour ces vilains résistants qui ont osé tuer sauvagement des officiers allemands? (rik\&rok, blog « Le Figaro », 18/10/2012);

17. La France ne cherche pas les causes de ses échecs actuels dans l'occupation allemande (troiscentsalheure, blog "Atlantico », 3/11/2012).

Dans ce contexte argumentatif, le recours à l'EH de l'Occupation peut aboutir à un jugement éthique de type nettement raciste, comme dans l'énoncé 18 , où la catégorie de ceux qui sont censés n'avoir besoin de demander aucune excuse (le «français blanc catholique ») est très clairement identifiée ${ }^{23}$ :

18) 18. Est-ce que tu as déjà vu un français blanc catholique pleurnicher et réclamer une reconnaissance de l'Allemagne pour tous les crimes commis pendant l'Occupation? (Kali, blog «Egalité et Réconciliation », 25/10/2012).

Utilisé en tant qu'argument de la pente fatale et appliqué à la question controversée du repentir public, l'EH peut agir aussi plus profondément, suggérant que l'histoire va dans le sens d'une dégénérescence, contre laquelle il ne serait plus possible de réagir qu'en revenant en arrière (c'est le schéma général de la pensée réactionnaire qu'a décrit Angenot 2004). Quelques interventions dans les blogs montrent ainsi qu'on peut être amené, suivant le schéma de la "mauvaise pente ", à rembobiner le fil de l'histoire et à remettre en cause la repentance archétypale, celle qu'a exprimée Chirac à propos de la déportation des Juifs et de la Shoah:

19. Elle [Marine Le Pen] ne dit pas que tout a commencé avec la repentance sur les deportations, et qu'a partir du moment qu'une minorité demande des comptes les autres vont faire pareil (Anonyme, blog « Egalité et Réconciliation », 25/10/2012) ; 
20. Je n'aime pas la repentance éternelle comme on subit depuis des lustres avec la shoah... ! (asmahane, blog « Egalité et Réconciliation », 25/10/2012).

\section{Conclusions}

L'observation des commentaires autour de la reconnaissance du massacre du 17 octobre 1961 nous a permis d'illustrer certains emplois de l'EH dans des contextes polémiques. Notre analyse a démontré la rentabilité de ce procédé, ainsi que la facilité avec laquelle il se prête, dans un corpus tel que le nôtre, à des constructions argumentatives qui vont bien au-delà des usages répertoriés dans la tradition rhétorique, comme l'argumentation par le précédent, la congérie, la diversion, la négation des faits traumatiques du passé, etc. Nous avons souligné notamment la disponibilité de l'EH à être mobilisé dans une stratégie comme celle de la "pente fatale », susceptible de connaître des usages caricaturaux, qui sapent la légitimité du débat par le recours à l'argument ad ridiculum. Cependant, certains aspects des EH que nous avons analysés méritent des considérations ultérieures, afin de saisir leur spécificité.

Une première réflexion concerne la notion même d'événement, vu comme une "structure à intelligibilité évolutive" (Rabatel 2014: 86). Soumis, comme plusieurs études l'ont démontré, à l'opacité du langage, l'événement est représenté tour à tour en tant qu'entité une et/ou multiple : il peut donc être pris en compte à lui seul, comme une donnée factuelle autonome (la guerre d'Algérie, la colonisation, l'Occupation, etc.) ou bien il peut être segmenté, inséré dans une série et orienté différemment, selon le positionnement axiologique et idéologique de l'énonciateur. La multiplicité des aiguillages possibles se retrouve et se renforce dans l'EH, qui est issu des événements : en effet, le même mécanisme peut servir des PDV ainsi que des ethè divergents, comme l'ethos du repentant et celui du non repentant.

Une deuxième considération porte sur les locuteurs ayant recours au procédé de l'EH dans les blogs. S'exprimant sous couvert d'anonymat, ces commentateurs relèvent d'une catégorie d'acteurs que la psychologie sociale identifie comme celui des « historiens naiffs, ou profanes» («the lay historian»: Klein 2013). Le statut du commentateur "profane " rejoint ainsi le citoyen sans compétences particulières, ou nettement inculte, à qui la rhétorique était finalement destinée depuis ses origines. La question de la représentation de l'événement historique de la part du citoyen ordinaire se pose donc ici, avec la constatation de la porosité des frontières séparant l'histoire (dans sa dimension de savoir spécialisé, autant que de savoir divulgué) et la mémoire, en tant qu'espace du monde vécu, tiraillé entre instance individuelle, subjective et instance groupale, collective (Hansson 2009, Ledoux 2013).

Une dernière réflexion concerne enfin, plus strictement, la nature de l'EH pouvant être exploité en tant qu'argument dans un contexte discursif polémique comme celui que nous avons observé. Tel que nous l'avons décrit, l'EH partage certaines caractéristiques avec le paradeigma (exemplum) historique classique, et notamment, l'ambiguïté foncière de son statut, oscillant entre occurrence singulière et figement en « type excellent » ou prototypique (Nicolas 2011), ainsi que la double appartenance aux mécanismes de l'induction et de la déduction (cf. Franchet d'Esperay 2013, Eggs 2014), puisque les faits singuliers tirent leur exemplarité de la relation qu'ils établissent avec des catégories 
générales (le «massacre", le «crime contre l'humanité », le "génocide », la "falsification historique ", la " repentance ", etc.).

Toutefois, deux caractéristiques au moins sont spécifiques aux EH que nous avons observés. La première est principalement d'ordre formel et catégoriel : se présentant sous la forme de l'allusion, l'EH que nous avons décrit acquiert quasiment un statut de figure figée, fonctionnant comme un stéréotype mémoriel, véhiculé par le simple nom d'événement, qui le transforme en «reliquat narratif» (Stierle 1972). Le nom propre, comme le recommandait Quintilien, occupe une place privilégiée dans les commentaires examinés, étant toujours mis en exergue, afin d'exploiter la charge interdiscursive avec laquelle ce nom est stocké en mémoire. De cette façon, l'EH devient le support d'une vision cristallisée du monde, d'un propos qu'on assène comme une évidence indiscutable, soustraite à la négociation, voire à la réfutation. En outre, la forme clichéique favorise une certaine autonomie de ces fragments mémoriels qui, dans leur circulation médiatique, sont facilement détachables et manipulables. Pour ces raisons, l'emploi de l'EH que nous avons observé s'apparente au paralogisme constitué par l'amalgame, tel qu'il a été décrit par Koren $(1995,2002)$.

31 La deuxième considération relève plutôt de la sémantique, ainsi que de cette « rhétorique profonde » à laquelle se réfèrent Danblon, Ferry, Nicolas et Sans (2014: 8). Issus davantage de la mémoire que de l'histoire, les exemples analysés ici affichent une nette propension à estomper la distinction que la tradition a toujours reconnue entre l'EH (en tant que fait avéré) et la fabula (fait inventé), comme le suggérait déjà Danblon $(2004)^{24}$. Les occurrences examinées se basent en effet sur une conception de la "vérité » historique qui tend à coïncider avec ce qu'une communauté parlante tient, revendique et met en scène comme étant le Vrai. Notre analyse touche donc, à la fois, à la question de la subjectivité, en tant que condition intrinsèque et inéluctable de tout discours (Koren 2008, 2012) et à la réflexion sur l'action des schémas narratifs primordiaux et mythiques, qui sont à l'œuvre dans le discours ordinaire (Danblon 2004, 2008). A cet égard, la contribution de ces schémas à la construction des identités collectives mériterait des approfondissements supplémentaires.

La convergence de tous les facteurs que nous venons d'énumérer (polysémanticité du nom de l'événement, statut de l'énonciateur en tant qu' "historien naïf », nature clichéique de l'EH de type allusif, fondement mémoriel du substrat événementiel) explique l'aptitude des EH de notre corpus à constituer des arguments éristiques, largement disponibles pour être mis à contribution dans des stratégies de persuasion volontiers caricaturales, comme celle de la "pente glissante». En effet, un corpus constitué des conversations numériques des lecteurs, visant à créer une " communauté virtuelle de protestation» (Amossy $2014: 193$ ), apparait tout particulièrement adéquat à démontrer que la "vérité " est une construction foncièrement rhétorique. Une réflexion de Nietzsche exprime efficacement cette idée, sur laquelle nous allons conclure :

Qu'est-ce que la vérité ? Une multitude mouvante de métaphores, de métonymies, d'anthropomorphismes, bref, une somme de relations humaines qui ont été poétiquement et rhétoriquement haussées, transposées, ornées, et qui, après un long usage, semblent à un peuple fermes, canoniales et contraignantes : les vérités sont des illusions dont on a oublié qu'elles le sont ${ }^{25}$. 


\section{BIBLIOGRAPHY}

\section{Corpus}

Béguin, François. 2012. « En condamnant la reconnaissance du 17 octobre 61, la droite soigne ses intérêts électoraux » (interview de Jean-Pierre Rioux), Lemonde.fr, 18 octobre, http:// www.lemonde.fr/politique/reactions/2012/10/18/en-condamnant-la-reconnaissance-du-17octobre-1961-la-droite-soigne-ses-interets-electoraux_1777763_823448.html\#(consulté le 20 février 2015)

Le Monde. 2012. « Le président, l'opposition et un massacre à Paris » (éditorial), Lemonde.fr, 19 octobre, http://www.lemonde.fr/a-la-une/article/2012/10/19/le-president-l-opposition-et-unmassacre-a-paris_1778167_3208.html (consulté le 20 février 2015)

Garat , Jean-Baptiste. 2012. « 17 octobre 61 : pour la droite, Hollande cherche à diviser », Lefigaro.fr, 18 octobre, http://www.lefigaro.fr/politique/

2012/10/17/01002-20121017ARTFIG00721-pour-la-droite-hollande-cherche-a-diviser-lesfrancais.php (consulté le 20 février 2015)

Perrault, Guillaume. 2012. « 17 octobre 1961 : Hollande fait repentance », Lefigaro.fr, 26 octobre, http://www.lefigaro.fr/politique/2012/10/17/01002-20121017ARTFIG00747-17-octobre-1961hollande-fait-repentance.php (consulté le 20 février 2015)

Cogné, Gaël. 2012. « Algérie. Les vraies raisons de la "repentance" de Hollande », Francetvinfo.fr ; 20 octobre, http://www.francetvinfo.fr/monde/afrique/algerie-les-vraies-raisons-de-larepentance-de-hollande_157885.html (consulté le 20 février 2015)

BFMTV. 2012. "Marine Le Pen : "A quand la repentance pour la Saint-Barthélémy ?" ", Bfmtv.com, 18 octobre, http://www.bfmtv.com/politique/marine-pen-la-corse-cest-france-361314.html (consulté le 20 février 2015)

Égalité-Réconciliation. 2012. « Marine Le Pen sur la repentance », egaliteetreconciliation.fr, 25 octobre, http://www.egaliteetreconciliation.fr/Marine-Le-Pen-sur-la-repentance-14534.html (consulté le 20 février 2015)

Atlantico. 2012. « La repentance: ce grand mal français » (interview de Dimitri Casali), atlantico.fr, 3 novembre, http://www.atlantico.fr/decryptage/repentance-grand-mal-francais-dimitricasali-533215.html (consulté le 20 février 2015)

Oster, Adrien. 2012. «"Excuses" : quels mots pour évoquer la guerre d'Algérie et la colonisation pour Hollande ? », huffingtonpost.fr, 19 décembre, http://www.huffingtonpost.fr/2012/12/19/ voyage-francois-hollande-algerie-repentance-excuses-guerre-colonialisme_n_2327914.html (consulté le 20 février 2015)

\section{Références}

Amossy, Ruth. 2000. L'argumentation dans le discours (Paris : Nathan)

Amossy, Ruth. 2014. Apologie de la polémique (Paris : PUF)

Angenot, Marc. 2004. Rhétorique de l'antisocialisme. Essai d'histoire discursive, 1830-1914 (Québec : Presses de l'Université Laval)

Angenot, Marc. 2012. « La notion d'arsenal argumentatif : l'inventivité rhétorique dans l'histoire ", Benoît Frydman \& Michel Meyer (éds), Chaïm Perelman. De la nouvelle rhétorique à la logique juridique (Paris : PUF), 39-68 
Angenot, Marc. 2014. «L'exemplum dans l'idéologie, ou : l'Affaire Dreyfus avant 1894 », Emmanuelle Danblon, Victor Ferry, Louis Nicolas \& Benoît Sans (éds). 2014. Rhétoriques de l'exemple. Fonctions et pratiques (Besançon : Presses universitaires de Franche-Comté), 39-59

Anthonissen, Christine. 2006. "The language of remembering and forgetting ", Journal of Language and Politics 5, 1-13

Chateauraynaud, Francis, Marianne Doury. 2013. « Le rôle des événements dans la portée des arguments : une affaire de 'précédents' ", Danielle Londei, Sophie Moirand, Sandrine ReboulTouré \& Licia Reggiani (éds), Dire l'événement : langage, mémoire, société (Paris : Presses Sorbonne nouvelle), 267-283

Danblon, Emanuelle. 2004. «L'exemple rhétorique : l'usage de la fiction en argumentation », Ruth Amossy \& Dominique Maingueneau (éds), L'analyse du discours dans les études littéraires (Toulouse : Presses Universitaires du Mirail), 187-198

Danblon, Emmanuelle, Ferry Victor, Loïc Nicolas \& Benoît Sans. 2014. « Introduction », Emmanuelle Danblon, Ferry Victor, Loïc Nicolas \& Benoît Sans (éds), Rhétoriques de l'exemple. Fonctions et pratiques (Besançon : Presses universitaires de Franche-Comté), 7-13

Danblon, Emmanuelle. 2008. « Fonctions éthiques et discursives de la narration. Un point de vue éclairé par la notion de rationalité discursive », Marie-Geneviève Pinsart (éd), Narration et identité. De la philosophie à la bioéthique (Paris : Vrin), 35-50

Derrida, Jacques. 2000. Foi et savoir (Paris : Seuil)

Dijk, Teun A. van. 1987. « Episodic models in discourse processing ", Rosalind Horowitz \& S. Jay Samuels, (éds), Comprehending oral and written language (New York : Academic Press), 161-196

Efe, Ibrahim \& Forchtner Bernhard. 2015, « 'Saying sorry'in Turkey. The Dersim massacre of the 1930s in 2011», Journal of Language and Politics 14, 233-257

Eggs, Ekkehard. 2014. «L'argument par l'exemple, l'exemplum et l'appropriation du passé. À propos des 'Justes' de France », Emmanuelle Danblon, Victor Ferry, Louis Nicolas \& Benoît Sans (éds), Rhétoriques de l'exemple. Fonctions et pratiques (Besançon : Presses universitaires de FrancheComté), 133-151

Forchtner, Bernhard. 2014. « Historia Magistra Vitae: The Topos of History as a Teacher in public struggles over self- and other representation », Christopher Hart \& Piotr Cap (éds), Contemporary Critical Discourse Studies (London : Bloomsbury), 19-43

Franchet d'Espèrey, Sylvie. 2010. «Le statut de l'exemplum historique chez Quintilien », PierreLouis Malosse, Marie Pierre Noël \& Bernard Schouler (éds), Clio sous le regard d'Hermès (Alessandria : Edizioni dell'Orso), 65-79

Hansson, Aleida. 2009. « From Collective Violence to a Common Future : Four Models for Dealing with a Traumatic Past », Ruth Wodak \& Gertraud Auer Borea (éds), 2009. Justice and Memory. Confronting traumatic pasts. An international comparison. (Wien : Passagen Verlag), 31-48

Hansson, Sten. 2015. « Discursive strategies of blame avoidance in government: A framework for analysis », Discourse \& Society 26, 297-322

Herman, Thierry. 2011. « De l'exemple à l'exemplum : réflexions sur leur efficacité rhétorique », Victor Ferry, Benoît Sans \& Alice Toma, « Etudes sur l'exemple », DICE, 8-2, 96-119

Klein, Olivier. 2013. « The lay historian : How Ordinary People Think About History», Rosa Cabecinhas \& Lilia Abedia (éds), Narratives and Social Memory: Theoretical and Methodological Approaches (Braga : University of Minho), 25-45 
Klinkenberg, Jean-Marie. 2000. «L'argumentation dans la figure », Cahiers de praxématique 35, 59-86

Koren, Roselyne. 1995. “'Concerning an 'argumentative monster': The perverted analogy in French journalistic discourse”, Frans H. van Eemeren, Rob Grootendorst, J.Anthony Blair \& Charles A. Willard (éds), Proceedings of the Third ISSA Conference on Argumentation, III, Reconstruction and application (Amsterdam : Sic Sat), 543-552

Koren, Roselyne. 2008. « Pour une éthique du discours : prise de position et rationalité axiologique ", Argumentation et Analyse du Discours 1, [En ligne : http://aad.revues.org/263]

Koren, Roselyne. 2012. « Langage et justification implicite de la violence : le cas de l"amalgame' ", Laurence Aubry \& Béatrice Turpin (éds). Victor Klemperer Repenser le langage totalitaire (Paris : Presses du CNRS), 93-105

Krieg-Planque, Alice. 2012. Analyser les discours institutionnels (Paris : Colin)

Labelle, Micheline, Rachad Antonius \& Georges Leroux (éds). Le devoir de mémoire et les politiques du pardon (Québec: Presses Universitaires du Québec)

Ledoux, Sébastien. 2013. «Les historiens face aux nouveaux usages du mot mémoire », Mots. Les langages du politique 103, 137-143

Lefranc, Sandrine. 2002. Politiques du pardon (Paris : PUF)

Maingueneau, Dominique. 1987. L'analyse du discours. Introduction aux lectures de l'archive, (Paris : Hachette)

Martin, James Robert \& Ruth Wodak (éds). 2003. Re/reading the past (Amsterdam : John Benjamins) Nicolas, Loïc. 2011. « L'exemple ambigü ou la phronèsis du phronimos », Victor Ferry, Benoît Sans \& Alice Toma, «Etudes sur l'exemple », DICE 8-2, 27-48

Paissa, Paola. 2012. «La gradualité de l'euphémisme : analyse d'un corpus de presse à la fin de la guerre d'Algérie », Marc Bonhomme, Mariela De La Torre \& André Horak (éds), Études pragmaticodiscursives sur l'euphémisme. Estudios pragmático-discursivos sobre el eufemismo, (Frankfurt am Main : Peter Lang), 175-192

Paissa, Paola. 2015. «L'hyperbole, une “figure dérivée” par excellence : revue des procédés rhétoriques d'hyperbolisation», Tranel 61-62, 25-41

Pelinka, Anton. 2009. « Justice, Truth and peace. Three Dimensions of Consequences », Ruth Wodak \& Gertraud Auer Borea (éds), Justice and Memory. Confronting traumatic pasts. An international comparison (Wien : Passagen Verlag), 49-65

Perelman, Chaïm \& Lucie Olbrechts-Tyteca. [1958] 2008. Traité de l'argumentation. La nouvelle rhétorique (Bruxelles : Éditions de l'université de Bruxelles)

Perelman, Chaïm. 1970. « Objectivité et intelligibilité dans la connaissance historique », Le Champ de l'argumentation (Bruxelles : Presses Universitaires de Bruxelles)

Quéré, Louis. 1994. "L'événement "sous une description” : contraintes sémantiques, croyances stéréotypiques et "natural facts of life as a morality" ", Protée 22, 14-28

Rabatel, Alain. 2005. « Les postures énonciatives dans la co-construction dialogique des points de vue : coénonciation, surénonciation, sousénonciation », Jacques Bres, Patrick Pierre Haillet, Sylvie Mellet, Henning Nolke \& Laurence Rosier (éds). Dialogisme, polyphonie: approches linguistiques (Bruxelles : Duculot) 95-110 
Sini, Lorella. 2015. «Événements, discours, médias : réflexions à partir de quelques travaux récents ", Argumentation et Analyse du Discours 14 [En ligne : http://aad.revues.org/1912]

Stierle, Karlheinz. 1972. «L'Histoire comme exemple, l'Exemple comme Histoire. Contribution à la pragmatique et à la poétique des textes narratifs », Poétique 10, 176-198

Tavukis, Nicholas. 1991. Mea Culpa. A sociology of Apology and Reconciliation (Stanford : SUP)

Turbide, Olivier, Marty Laforest \& Diane Vincent (éds). 2013. « Le repentir public comme mode de gestion de crise. Quelques stratégies d'atténuation de l'offense et de la responsabilité de l'offenseur », Raphaël Micheli, Ida Hekmat \& Alain Rabatel (éds), « Les émotions argumentées dans les médias ", Le discours et la langue 4.1,137-157

Walton, Douglas Neil. 1992. Slippery Slope Arguments (Oxford : Clarendon Press).

Wodak, Ruth \& Gertraud Auer Borea (éds). 2009. Justice and Memory. Confronting traumatic pasts. An international comparison (Wien : Passagen Verlag)

Wodak, Ruth. 2006. « Blaming and Denying : Pragmatics », Keith Brown (éd.), Encyclopedia of Language and Linguistics, $2^{\text {nd }}$ ed., vol.2 (Oxford : Elsevier), 59-64

Wodak, Ruth. 2008. «The contribution of critical linguistics to the analysis of discriminatory prejudices and stereotypes in the language of politics ", Ruth Wodak \& Veronika Koller (éds), Handbook of Communication in the Public Sphere (Berlin, New York : Mouton de Gruyter), 291-315

Wodak, Ruth. 2015, The politics of fear. What right-wing populist discourses mean (London : Sage)

\section{NOTES}

1. Le communiqué de la Présidence relève du discours institutionnel et, plus précisément, pour sa nature performative, de l'acte de langage du type « excuses d'Etat » - cf. Krieg-Planque 2012 : 74. Sur l'analyse d'un cas de " political apology » en tant que « speech act», voir Efe et Forchtner 2015.

2. La liste complète de ces articles, parus dans des quotidiens ou sites divers, figure en bibliographie.

3. L'«argument de la direction» a donné lieu à plusieurs désignations métaphoriques : argument de la «pente glissante ", de la «pente fatale ", de la «pente savonneuse ", du « doigt dans l'engrenage», etc. Dans la suite de notre article, nous utiliserons donc ces appellations comme équivalentes et interchangeables.

4. La présence d'éléments de ce genre est considérée comme un critère caractérisant le discours mettant en scène le « repentir public » (cf. Tavuchis 1991 ; Turbide, Laforest et Vincent 2012).

5. Il s'agit d'une figure de personnification : cf. Perelman et Olbrechts-Tyteca $2008: 445$.

6. Pour un état présent de la bibliographie sur la nomination des événements, fort vaste désormais, nous renvoyons à Sini 2015.

7. Le mot s'applique à la sphère politique et juridique mais appartient, tout autant que "pardon », au discours religieux (et, plus précisément, " abrahamique », suivant Derrida 2000). Sur la question, cf. aussi Lefranc 2002 et Labelle, Antonius et Leroux 2005.

8. La description de l'emploi de l'EH débouche évidemment sur la question plus générale des modalités de la narration du passé dans le discours public. Dans la riche bibliographie sur cette question, formant l'objet principal de la DHA (Discourse Historical Approach), nous renvoyons à Martin et Wodak 2003, Anthonissen 2006, Wodak et Aurea Borea 2009.

9. Il s'agit de la stratégie argumentative jouant sur l'opposition de l'acte et de l'essence (cf. Perelman et Olbrechts-Tyteca 2008: 467-468). Suivant l'optique de la presse algérienne, la 
reconnaissance de certains épisodes isolés (actes) ne permettrait pas de saisir l'essence de la faute (la guerre, la colonisation).

10. Dans le quotidien algérien Liberté du 18/10/2012, repris dans Courrier International.

11. C'est la stratégie de négation et de justification s'appuyant sur le cliché «Others are no better : War is War » : cf. Wodak $2006: 62$.

12. Il est toutefois connu qu'une différence d'ordre quantitatif dans ce genre de corpus n'est pas significative en elle-même, les membres d'une discussion en ligne réagissant d'habitude plus facilement pour critiquer que pour approuver l'objet d'un débat. En outre, la situation de communication informelle des blogs favorise, comme d'habitude, la formulation de contenus injurieux, discriminatoires et relevant parfois ouvertement du « syncretic racism » (Wodak 2008, Amossy 2014).

13. Perelman, suivant Bacon, désigne les exemples « rapportés succinctement » comme ceux qui relèvent d'un usage " servile ", s'opposant à l'« usage inductif » qui, en revanche, exigerait des « exemples détaillés » : Perelman et Olbrechts-Tyteca $2008: 482$.

14. Suivant la rhétorique du « juge-pénitent » qu'illustre Forchtner, la reconnaissance de la faute légitimerait finalement une attitude de supériorité morale (voir l'article de B. Forchtner, dans le présent numéro).

15. Dans les exemples ont été conservés, comme de coutume, l'orthographe souvent fautive et les marques typographiques du commentaire original. Pour faciliter nos renvois, nous avons numéroté tous les commentaires rapportés, indépendamment du fait qu'ils contiennent des $\mathrm{EH}$ ou qu'ils soient simplement destinés à illustrer les enjeux polémiques des deux camps.

16. Ces commentaires ont été publiés respectivement dans les blogs du Huffington Post.fr, 19/10/2012 et du Figaro, 18/10/2012.

17. Pour une définition et une description des modalités d'usage du "précédent", nous renvoyons à Chateauraynaud, Doury 2013.

18. En effet, suivant Pelinka 2009, la situation asymétrique des deux parties en conflit et le manque d'une phase de recherche de la vérité par voie judiciaire, rendraient impossible une véritable réconciliation, comme les commentaires analysés ici le montrent bien.

19. En effet, dans le commentaire 7, le crime commis par la France est relativisé, alors que deux faits sont reprochés au FLN, avec une utilisation non distanciée, la deuxième fois, du mot "massacre ", s'accompagnant de l'utilisation du mot "victimes » et de l'insistance sur la durée des fautes de l'Algérie, envers les harkis, se prolongeant jusqu'à l'époque actuelle. Dans 7, on ne se contente pas de renvoyer la France et l'Algérie dos à dos, mais on suggère aussi que la deuxième est « plus coupable » que la première.

20. Il s'agit ici de la stratégie, considérée comme fallacieuse, du changement de sujet (trajecto in alium): on compare des objets trop éloignés et différents, ouvrant une fausse piste au raisonnement (stratégie de la diversion, dite aussi du « hareng rouge »), cf. Hansson 2015.

21. Sur un cas semblable d'utilisation de l'hyperbole, cf. Paissa 2015.

22. Dans ce cas de figure, on pourrait parler également d'argument par l'absurde. Cependant, nous suivrons, une fois de plus, Perelman et Olbrechts-Tyteca 2008: 276-282), qui préfèrent réserver la notion d'absurde au plan de la logique (contradiction, incompatibilités, etc.), tout en soulignant que le ridicule « joue dans l'argumentation un rôle analogue à celui de l'absurde dans la démonstration $»(280)$.

23. Alors que les propos racistes sont souvent plus génériques, relevant du syncretic racism, « racisme sans race» (Wodak 2008: 293), comme cela arrive dans le discours populiste.

24. Pour des considérations sur l'évolution de cette distinction, cf. Stierle 1972.

25. La citation de Nietzsche (Le livre du philosophe, Paris, Aubier-Flammarion, 181-182), empruntée à Derrida, est rapportée dans Klinkenberg (2000:86). 


\section{ABSTRACTS}

This paper analyzes a corpus of readers' comments published in newspapers' talkbacks. The corpus involves the readers' responses to the October $17^{\text {th }} 2012$ press release in which President Hollande acknowledged the massacre of Algerian demonstrators that took place on October 17th in Paris, a few months before Algeria's independence. In particular, the article examines how bloggers use the historical example as a polemical rhetorical tool aimed at criticizing the President's initiative. In this view, the historical example participates in a form of political apology triggering conflicting memories. In the corpus analyzed, it is often used fallaciously in order to deny either past wrongdoings, or the responsibility of the French State, or even in order to establish dubious comparisons with other traumatic past experiences. In this framework, the historical example is also used as a typical device for constructing slippery slope arguments and ironic rhetorical questions, thus contributing to build an ad ridiculum argumentative discourse.

L'article analyse l'emploi de l'exemple historique (EH) dans un corpus de commentaires de lecteurs, publiés dans les forums en ligne d'un certain nombre de quotidiens. Ces commentaires réagissent au communiqué du 17 octobre 2012, par lequel le Président Hollande a reconnu le massacre de manifestants algériens qui avait eu lieu à Paris le 17 octobre 1961, quelques mois avant l'Indépendance de l'Algérie. Le procédé de l'EH est utilisé par les blogueurs en tant qu'argument polémique, qui a généralement pour finalité de critiquer l'initiative de la Présidence. Celle-ci s'encadre, quant à elle, dans le paradigme discursif de la repentance publique et réactive, par conséquent, des réseaux mémoriels controversés. Dans les blogs examinés, l'EH est donc exploité de manière volontiers paralogique ou fallacieuse, pour désavouer in toto le méfait du passé, pour nier la responsabilité de l'Etat français ou encore pour établir des comparaisons douteuses avec d'autres expériences historiques traumatisantes. Dans ce cadre, une place importante est réservée à l'emploi de l'EH dans le schéma argumentatif de la «pente glissante » et dans l'interrogation rhétorique de type ironique, un dispositif s'avérant fonctionnel pour la mise en place d'une argumentation ad ridiculum.

\section{INDEX}

Keywords: fallacy, historical example, October 17th 1961 massacre, polemical tool, political apologies, slippery slope argument

Mots-clés: argument de la pente glissante, argument fallacieux, exemple historique, massacre du 17 octobre 1961, polémique, repentance

\section{AUTHOR}

PAOLA PAISSA

Université de Turin 